\title{
Metodologías en reintegraciones volumétricas de prefabricados con carácter decorativo
}

\author{
Methodologies in volumetric reintegration of prefabricated \\ elements with decorative character
}

\section{Resumen}

Autores:

Juan Bermejo-Soler* juan.bermejo@ehu.eus Iñigo González-González* inigo.gonzalezg@ehu.eus Amaia Conde-Chiralt** accescultura@gmail.com

Nagore Prieto-Taboada* nagore.prieto@ehu.eus

Fernando Baceta-Gobantes*

fernando.bazeta@ehu.eus Ma. Dolores Rodríguez-Laso* mariadolores.rodriguez@ehu.eus Juan Manuel Madariaga-Mota* juanmanuel.madariaga@ehu.eus

* Universidad del País Vasco **ACC Técnicas de escultura

\section{T-}

os elementos decorativos prefabricados en Patrimonio Construido tienen una gran importancia estética, sobre todo en las edificaciones de finales requieren de una intervención de rehabilitación integral, así como también sus elementos decorativos. Es el caso del proyecto de recuperación y puesta en valor de las galerías Punta Begoña (Getxo, España), fruto de la colaboración de Ayuntamiento de Getxo y la Universidad del País Vasco UPV/EHU, se están desarrollando nuevas metodologías, a partir de los sistemas de moldeo existentes, para mejorar las intervenciones de restauración que se realizan en este campo, así facilitar el citado trabajo y con ello permitir el respeto al máximo de los elementos originales conservados. Actualmente se están efectuando trabajos con tres variaciones sobre los moldes elásticos laminados, lo que permite realizarlos sin contacto directo con la obra e intentando reintegrar fragmentos originales en su interior.

Palabras clave: patrimonio construido; Punta Begoña; molde; laminado; reintegración.

\section{Abstract:}

The precast decorative elements found in Built Heritage have a great aesthetic importance, especially in the late nineteenth and early twentieth centuries. At the moment, many of these buildings are in need of a full rehabilitation intervention, as well as its decorative elements. As part of the recovery and enhancement project of the Punta Begoña galleries (Getxo, Spain), outcome of the collaboration between Getxo City Council and the University of the Basque Country UPV / EHU, new methodologies are being developed, based on existing molding systems, to improve restoration interventions carried out in this area, and thus ease the work and provide the maximum respect to the conserved original elements. Currently, work is being executed with three variations of the laminated elastic molds, which allows them to be applied without direct contact with the artistic element and reintegrating missing parts of original fragments inside the mold.

Keywords: Built Heritage; Punta Begoña; mold; laminate; reintegration. 


\section{Introducción}

La restauración de patrimonio construido presenta una gran complejidad y requiere la continua investigación y formación de todo e personal que participa en ella. La dificultad se acrecienta cuando se trata de patrimonio más reciente, debido a los grandes cambios que se produjeron a lo largo del siglo XIX y XX, con la aparición de nuevos materiales y técnicas constructivas, innovadoras para la época. Por todo lo expuesto, se propone, desde este trabajo, optimizar las metodologías actuales para la recuperación de elementos decorativos prefabricados en mortero y hormigón.

A partir de la segunda mitad del siglo XIX, con el descubrimiento del cemento artificial y la introducción del hormigón armado, se generalizó el uso de este tipo de elementos, teniendo una mayor participación con un fuerte peso estético en la arquitectura de la época. Habitualmente, estos elementos se ubicaban en las fachadas y cerramientos, por lo cual se extendió su uso como partes constituyentes de balaustradas, molduras, suelos, etc.

Este trabajo nace a raíz del proyecto de "Recuperación y puesta en valor de las Galerías Punta Begoña", el cual tiene un interés arquitectónico destacable y está situado en el municipio de Getxo (Bizkaia, España). Esto se debe a la gran importancia de las innovaciones técnicas utilizadas en la construcción de las galerías, a su calidad artística, e incluso al recorrido profesional de sus promotores, así como a la gran cantidad de sucesos ocurridos en su interior.

Punta Begoña es una edificación al borde de mar, compuesta por dos grandes galerías que forman un ángulo aproximado de 90ㅡ, con un jardín en la parte superior que tiene el cometido de techo de las citadas galerías (Figura 1). La primera, entre ellas, mira a mar abierto, con una dirección NW, en forma de S, y una longitud de unos 100 m., al final de la cual encontramos una gran estancia y un par de espacios que comunican con el citado jardín mediante una escalera. La galería SW, con un total de 90 m., da a la zona de la bahía del Abra, formada, en su inicio, por un tramo curvo, dos tramos rectos, y un espacio circular o tholo que conecta en su parte superior con la galería NW ya mencionada.

Horacio Echevarrieta, quien fuera un importante empresario de la zona, encarga la construcción de un palacete para su familia al arquitecto Gregorio Ibarretxe en 1910. Este palacete se encontraba sobre el acantilado de Arriluce, lo que obliga al propietario a dar una solución a los problemas de desprendimientos procedentes de las rocas que forman el citado acantilado.

La solución viene de la mano del arquitecto Ricardo Bastida, quien en 1918 diseña el proyecto y dirige la ejecución de las galerías Punta Begoña, para lo cual no realiza un simple muro de contención para el acantilado, sino también un belvedere, es decir, una zona para la contemplación de las vistas de cara al mar. Además, se busca crear un icono en el paisaje, muestra de la importancia social y estatus económico en el que se movía la familia.

El conjunto se edificó entre 1918-1922, y se convierte en el primer edificio residencial de Bizkaia en utilizar el hormigón armado como elemento estructural (EI conjunto se edificó entre 1918-1922, y se convierte en el primer edificio residencial de Bizkaia en utilizar el hormigón armado como elemento estructural (Bermejo et al., 2018; Arroita et al., 2020).

Esta época de disfrute terminó pronto ya que, con el comienzo de la Guerra Civil Española (1936-1939), la propiedad al completo fue convertida en un hospital de convalecencia, dentro del sistema de hospitales de guerra diseñado por José María Bengoa. Este escogió

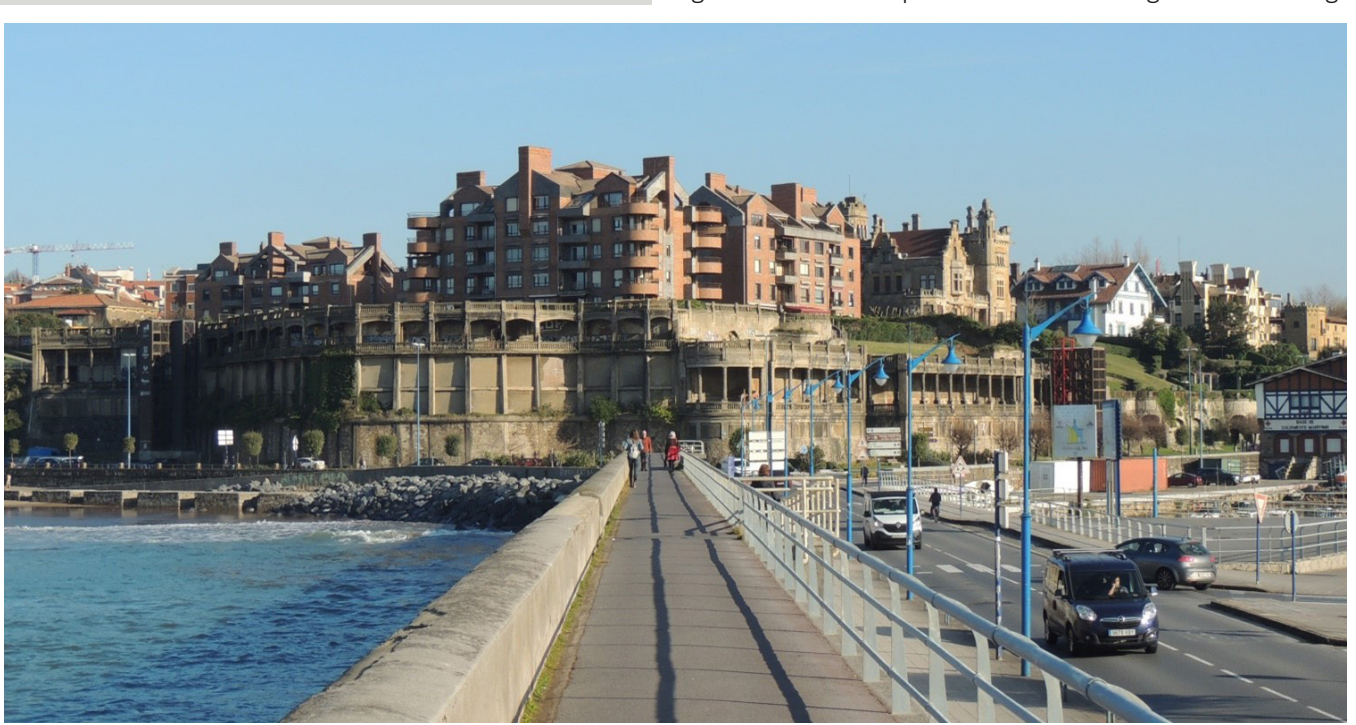

Figura 1: Vista general de las Galerías Punta Begoña. Paseo de Ariluce, Getxo, España

Fuente: Grupo B.B.A.A. (2019) 
Metodologías en reintegraciones volumétricas de prefabricados con carácter decorativo

la ubicación por estar alejada del frente y tener gran ventilación, lo que ayudaría a los heridos a su pronta recuperación por las condiciones climáticas de la zona (Gondra, 2018).

Tras la caída de Bilbao en junio de 1937, el complejo arquitectónico se convirtió en el cuartel del mando italiano para pasar, con el tiempo, a ser sede del Auxilio Social, controlado por la Sección Femenina de la Falange (Lama, 2020).

Finalmente, la propiedad fue devuelta a la familia Echevarrieta. Tras la muerte del patriarca, en 1963, esta se vendió y, en 1976, los edificios superiores fueron demolidos: el templete de acceso a la galería NW, la casa del guardes, así como el propio palacete. Como resultado de varias negociaciones con el Ayuntamiento de Getxo, entre 1988-1992 se construyó la urbanización que existe, actualmente, en el espacio ocupado por los edificios mencionados después de su desaparición (Bermejo, 2019).

Tras un siglo de exposición a todo tipo de agentes de deterioro: agua, contaminantes atmosféricos, aerosoles marinos y varios cambios de uso, el conjunto ha sufrido grandes daños, no solo a nivel estructural sino en los elementos que lo decoraban. En los distintos materiales (morteros, esgrafiados, pinturas murales, mármoles y cerámicas) hay problemas de fracturas, perdidas, descohesión, colonización biológica, costras cálcicas, depósitos de contaminantes y diversos actos vandálicos.

En 2013 comenzó su puesta en valor por parte de Ayuntamiento de Getxo y la UPV/EHU. Al presente varios grupos de investigadores de la universidad

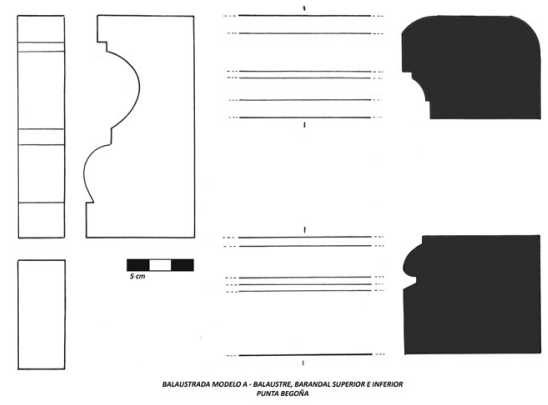

trabajan de manera transdisciplinar en su recuperación Hidrogeología (HGI), Arqueología (GPAC), Química Analítica (IBEA), Cátedra UNESCO y el grupo de Conservación y Restauración de la Facultad de Bellas Artes.

El uso principal de los prefabricados en Punta Begoña ha sido para construir las balaustradas que sirven de cierre de las distintas zonas y sus accesos. Actualmente se conocen tres modelos de balaustradas con sus correspondientes balaustres, barandales superiores $e$ inferiores.

Modelo A se ha denominado a la balaustrada que recorre la fachada a lo largo de todo el conjunto. Está compuesto por un barandal inferior, fabricado en hueco y rellenado en obra; un balaustre inscrito en un prisma de base rectangular, y dos modelos de barandal superior, uno recto y otro curvo. Dicho modelo solo presenta decoración por la cara externa que da a la fachada (Figura 2)

El modelo B sirve de cerramiento a la escalera que conecta el jardín superior de la galería NW con la terraza situada por encima de la galería SW. Es similar en disposición y estética al modelo A, pero a una escala más reducida. Además, algunos de los balaustres contaban con una base en ángulo para adaptarse a los tramos ascendentes (Figura 3).

El modelo $\mathrm{C}$ corresponde a las escaleras de acceso a la galería SW. Tiene un barandal inferior macizo y decorado por ambas caras, un balaustre formado por un objeto de rotación, con una base en ángulo y un barandal superior, igualmente decorado por ambas caras (Figura 4).

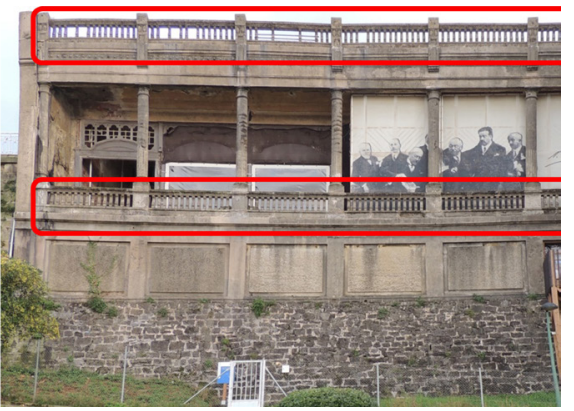

Figura 2: Esquema del modelo A, y primer tramo de la fachada en el que se aprecia Fuente: Grupo B.B.A.A. (2020)
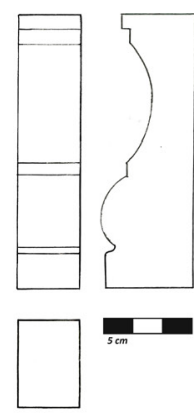

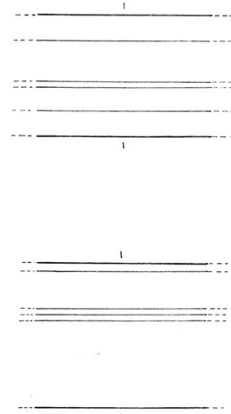

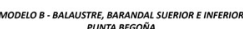
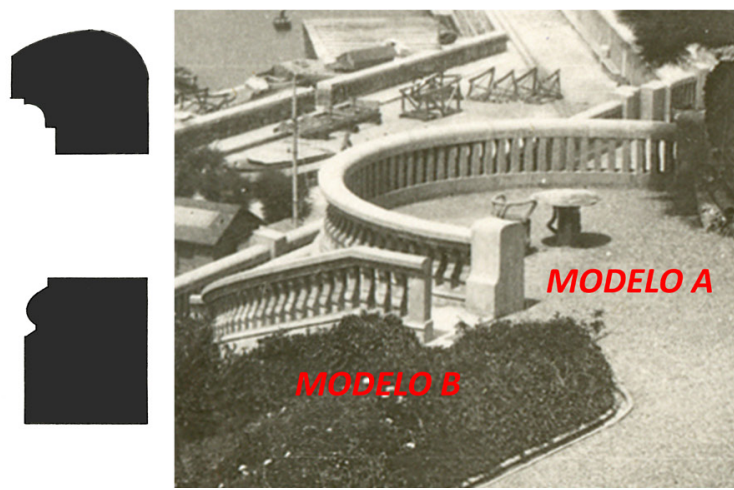

Figura 3: Esquema del modelo B, y fotografía antigua en la que se aprecia parte de este

Fuente: Izquierda: Grupo B.B.A.A. (2019). Derecha: GPAC y Diputación Foral de Bizkaia (Anterior a 1976) 

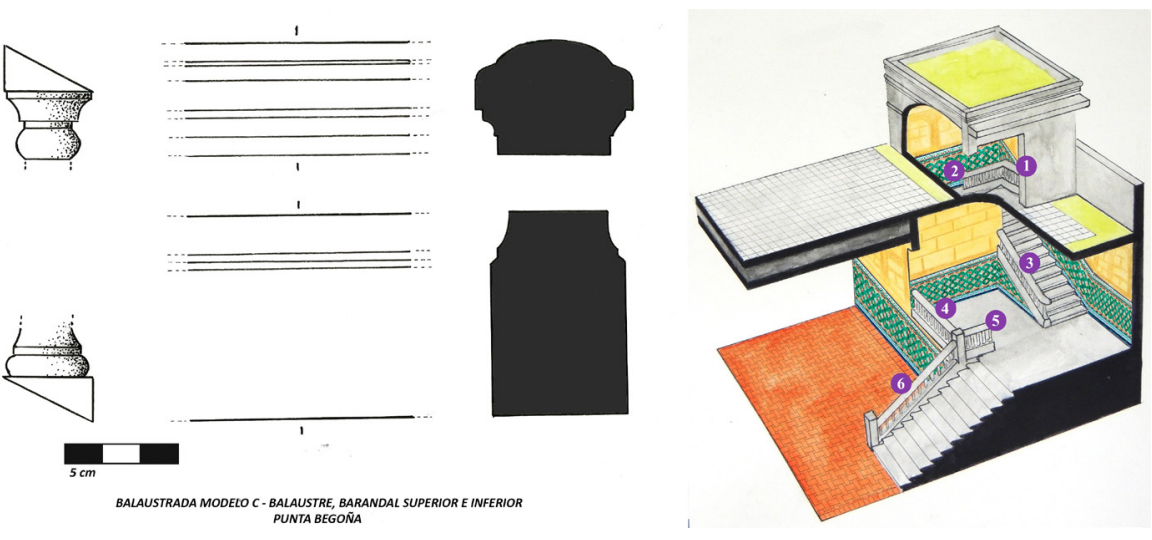

Figura 4: Esquema del modelo C, e ilustración de la zona en que se encuentra

Fuente: Grupo B.B.A.A. (2019-2020)

Todas las piezas están realizadas con morteros de cemento cuyos análisis nos han permitido conocer mejor los materiales utilizados originalmente, así como evaluar los procesos de degradación a que están sometidos. En trabajos anteriores se han empleado diversas técnicas de análisis, tanto estructural, molecular, como elemental, lo que nos ha permitido saber que utilizaron como conglomerante, al menos en parte, cemento portland y, como árido, arena de playa (Bermejo, 2019).

Con respecto a las armaduras encontramos diferentes opciones: el modelo A, cuenta con armaduras solo en el barandal superior; los modelos B y C llevan una armadura en los balaustres, pero no así en los barandales. En ningún caso los refuerzos metálicos se conectaban entre sí, ni con los elementos estructurales del edificio, lo que reduce mucho la capacidad de absorción de los movimientos en las balaustradas. No obstante, las ventajas de unir las armaduras entre sí no se conocían en el momento de edificación de las galerías (Calvo, 2016). La unión entre las piezas se ha realizado de diferentes maneras, dependiendo de las necesidades, mediante el uso de anclajes metálicos o rellenos de mortero. Por último, los modelos A y B se revistieron por completo con un mortero de enlucido.

Se desconoce el fabricante de las balaustradas, pero tal vez fuera la Compañía Anónima del Hormigón Armado de Sestao, la cual, en 1905, comenzó a fabricar este tipo de elementos ornamentales, como las balaustradas de Establecimiento de baños del Sardinero en Santander, o los remates de las chimeneas del Santo Hospital Civil de Bilbao (Basurto). Esta teoría es apoyada por la conocida colaboración de dicha empresa con Ricardo Bastida, autor de las galerías, en otros proyectos como la Alhóndiga, el lavadero modelo de Alameda San Mames, o los elementos decorativos de las Nuevas Escuelas Municipales de Bilbao (Burgos, 2009).

Otra posibilidad sería la Constructora Bilbaína, la cual se encargó de la fabricación de las baldosas prefabricadas de la parte superior de la galería SW. Dicha opción se vería apoyada por la fecha de construcción de las galerías (1918-1922), en las cuales la Compañía Anónima del Hormigón de Sestao se comenzaba a centrar más en la fabricación de cemento que en las obras de construcción (Burgos, 2009).

\section{Los moldes y su uso en patrimonio}

En patrimonio construido, ocasionalmente es necesario reproducir elementos faltantes por tener una función práctica, lo cual es más sencillo en el caso de los elementos seriados, ya que podemos conocer su aspecto original con mucha más facilidad.

El uso de moldes es ideal para la reproducción de elementos prefabricados, no solo por el acabado final, si no por su similitud a la técnica original de creación.

Se denomina molde a toda pieza, o conjunto de ellas, en las que se genera un hueco para, posteriormente, rellenarlo del material que se desee (Navarro, 2011). Los sistemas de moldeo tradicionales son de tipo directo, es decir, se realizan sobre la pieza original. Esto puede suponer ciertos riesgos para la obra, tanto por su manipulación como por la impregnación permanente de algunos de los materiales que constituyen los moldes (Mas, 2006). Por lo tanto, en este trabajo se propone el uso complementario de técnicas indirectas, lo que permite realizar reproducciones de calidad sin poner en riesgo la pieza original.

Dentro de las técnicas de reproducción directas, dependiendo de las características de los materiales en los que se realicen, podemos agruparlos en moldes rígidos o moldes elásticos (Figura 5). Los moldes rígidos, prácticamente en desuso para intervenciones en patrimonio construido, se basan en la realización de un molde en yeso. En el caso del molde perdido: se modelaba la pieza deseada en arcilla o cera; se ejecutaba el molde y se extraía la cera o el barro para rellenarlo con el material definitivo. Una vez terminado se destruía el molde de yeso para extraer el positivo.

En el molde rígido por piezas se subdividía la superficie del original en pequeñas zonas que no presentaran contrasalidas, creándose un negativo de yeso para cada una de ellas. Estos negativos se ensamblaban entre sí, permitiendo extraer el original y reutilizar el molde varias veces. Esta técnica se ha usado, tradicionalmente, para la realización de reproducciones en yeso de piezas clásicas. 


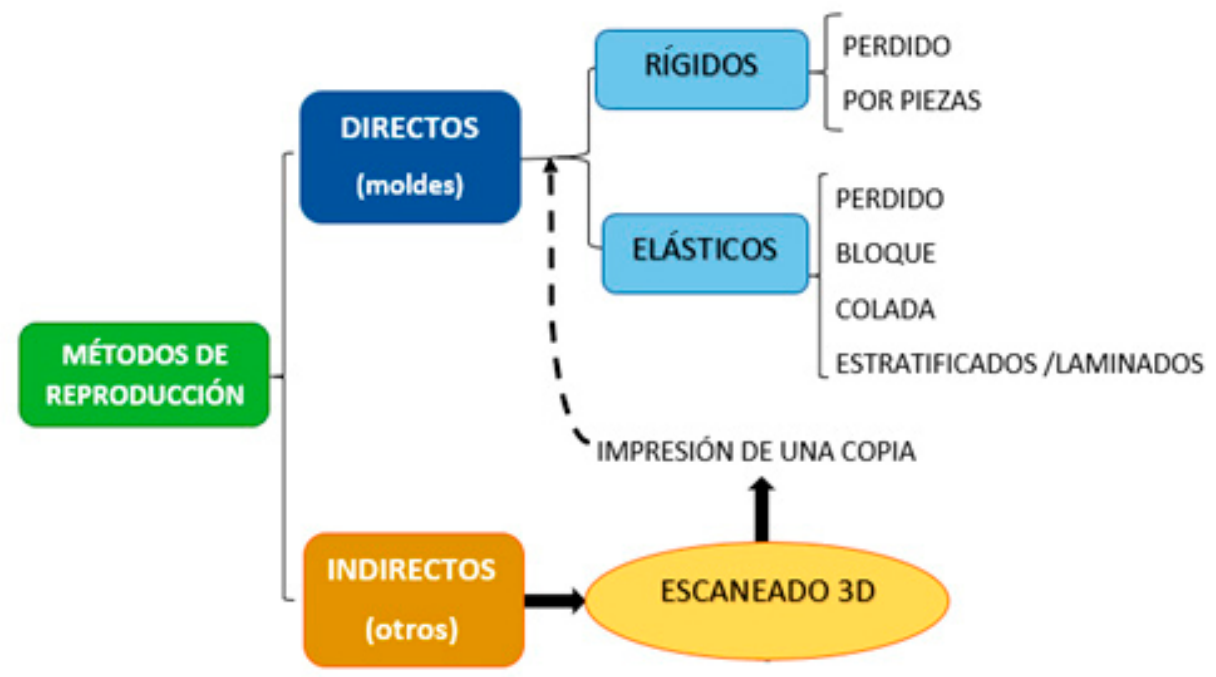

Figura 5: Esquema de los diferentes métodos de reproducción

Fuente: Grupo B.B.A.A. (2020)

En contadas ocasiones se usan, para intervenciones en patrimonio construido, moldes rígidos por piezas, sustituyendo el yeso por fibra de vidrio y resina de poliéster. Estos moldes permiten la manufactura de un elevado número de positivos, pero no son demasiado prácticos cuando se trata de elementos complejos.

Los moldes elásticos se basan en el uso de polímeros como el látex o las siliconas. Según el diseño del molde se distinguen tres tipos de moldes: bloque, colada o laminado.

El molde de bloque es el más sencillo de realizar, pero solo se puede utilizar con piezas que no presenten contrasalidas y de un tamaño relativamente pequeño. Este sistema consiste en fijar la pieza a la base de un recipiente y rellenarlo con silicona. Cuando esta cataliza se elimina el recipiente y se extrae el original por la zona de la base.

Los moldes de tipo colada son los que permiten hacer piezas más complejas, pero también son los más difíciles de elaborar. Permiten trabajar con piezas pequeñas y medianas. A grandes rasgos, consiste en aplicar una capa de plastilina o arcilla sobre la pieza a reproducir, y sobre esta crear un contramolde en yeso o fibra de vidrio. Una vez endurecido se elimina la plastilina o la arcilla y se vierte silicona en el espacio que queda entre el contramolde y el original.

Los moldes laminados o estratificados son, con diferencia, los más utilizados en la reproducción de elementos en patrimonio construido, por su gran versatilidad y permitir trabajar sin tener que mover la pieza de su posición. Este tipo de moldes son ideales para trabajos in situ y para piezas medianas y grandes.

El proceso de laminado consiste en aplicar, sucesivamente, diferentes capas de silicona o látex, hasta conseguir el espesor deseado; después se añaden al menos dos capas de fibra de vidrio y una resina para generar el contramolde.

\section{Métodos}

En las galerías Punta Begoña se recurrió, en gran medida, a la utilización en la decoración de elementos prefabricados de mortero, tanto armado como en masa, habiéndose registrado 36 piezas distintas. En este trabajo se describen exclusivamente los que forman las balaustradas. Primeramente, se realizó una descripción in situ de las distintas piezas y su ubicación. Posteriormente se estudió su estado de conservación, a raíz del cual se constató que el principal problema que sufren es la perdida de elementos.

Los tres modelos de balaustradas, debido a su estado de conservación y a la premisa de conservar el material original al máximo posible, necesitaron tres propuestas diferentes. En el primer caso se optó por realizar un molde laminado con la técnica habitual, pero los otros dos requirieron experimentar nuevas técnicas.

En el modelo B solo se conserva una pieza original, por lo que nos decantamos por una técnica de moldeo indirecta; se apoyó en el uso de la fotogrametría y la impresión 3D FDM.

Del modelo $\mathrm{C}$ solo se conservaban fragmentos, lo que hizo seleccionar un sistema de moldeo que permitiera introducir los fragmentos originales antes del vaciado, quedando de nuevo en su posición.

Para la realización de los moldes se recurrió a la técnica del laminado, aplicando tres capas de silicona Silastic 3481 con su catalizador al 5\%, y un aditivo tixotrópico $(<5 \%)$ en los casos que se consideró necesario. El contramolde se realizó con fibra de vidrio cuatriaxial y resina acrílica Jesmonite AC100. 


$\begin{array}{r}\text { Juan Bermejo-Soler / Iñigo González-González / Amaia Conde-Chiralt / Nagore Prieto-Taboada / Fernando } \\ \text { Baceta-Gobantes / Ma. Dolores Rodríguez-Laso / Juan Manuel Madariaga-Mota }\end{array}$
Metodologías en reintegraciones volumétricas de prefabricados con carácter decorativo

Para el levantamiento fotogramétrico se utilizó una cámara Nikon Coolpix P510, y un pequeño set fotográfico portátil. En el tratamiento de las imágenes se recurrió a los softwares VisualSFM (con las herramientas complementarias PMVS/CVMS de Yasutaka Furukawa's), Meshlab 2016.12 y Blender 2.8.

El proceso de impresión 3D se realizó con una impresora FDM Creality Ender 3, con un filamento de PLA de la casa comercial Tianse. Para preparar los archivos se utilizó el software de laminado Ultimaker Cura 4.4.

\subsection{Molde laminado convencional}

En primer lugar, se ha seleccionado uno de los balaustres sueltos que se encuentra completo; seguidamente se le ha aplicado desmoldante y, a continuación, se le ha dado una primera capa de silicona Silastic 3481 mezclada al 5\% con su catalizador. Una vez endurecida esta primera capa, se le han añadido otras dos manos de silicona, esta vez con un agente tixotrópico. En la aplicación de la tercera capa se han colocado unos prismas de silicona, que harán de separación entre las partes del contramolde y facilitarán la unión entre ambos. Posteriormente, se les ha sumado dos capas de resina acrílica Jesmonite AC100 reforzada con trozos de fibra de vidrio cuatriaxial. Una vez catalizada se han realizado los agujeros para los tornillos que mantendrán unidas las distintas partes del contramolde. Finalmente, se ha extraído el original y positivado el molde (Figura 6).

\subsection{Moldeo indirecto}

En el caso del balaustre del modelo $\mathrm{B}$, al conservarse tan solo una pieza completa, se ha optado por el uso de una técnica de reproducción indirecta, para no arriesgar el único original que se conserva. Para ello se realizó un levantamiento fotogramétrico de la pieza conservada. En primer lugar, con el apoyo de un pequeño equipo de iluminación se fotografió desde diferentes ángulos. Posteriormente, con diversos softwares se generó un levantamiento fotogramétrico de la pieza; a continuación, se posicionaron las fotografías, se generó una nube de puntos densa $y$, después, una malla. Se cerraron las aberturas que quedaban en la malla y se dividió la pieza por la mitad. Esta división responde al espacio de trabajo de la impresora, de la que dispongamos, pudiendo hacerse tantas partes como sea necesario. Después, los diferentes fragmentos se transforman, mediante el software de laminado para, seguidamente, proceder a su impresión.

Una vez impresas las distintas partes se unen mediante su encolado. A partir de este elemento se realizó un molde elástico laminado, tal y como se ha descrito en el caso anterior (Figura 7).

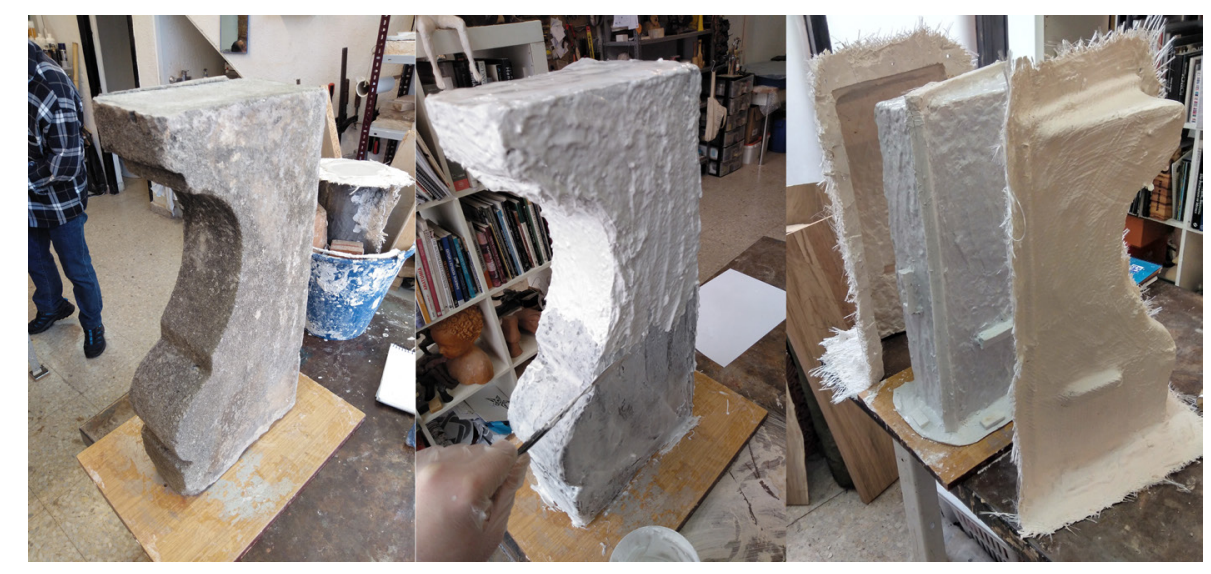

Figura 6: Proceso de realización de un molde laminado convencional para el balaustre del modelo A Fuente: ACC Escultura (2018)
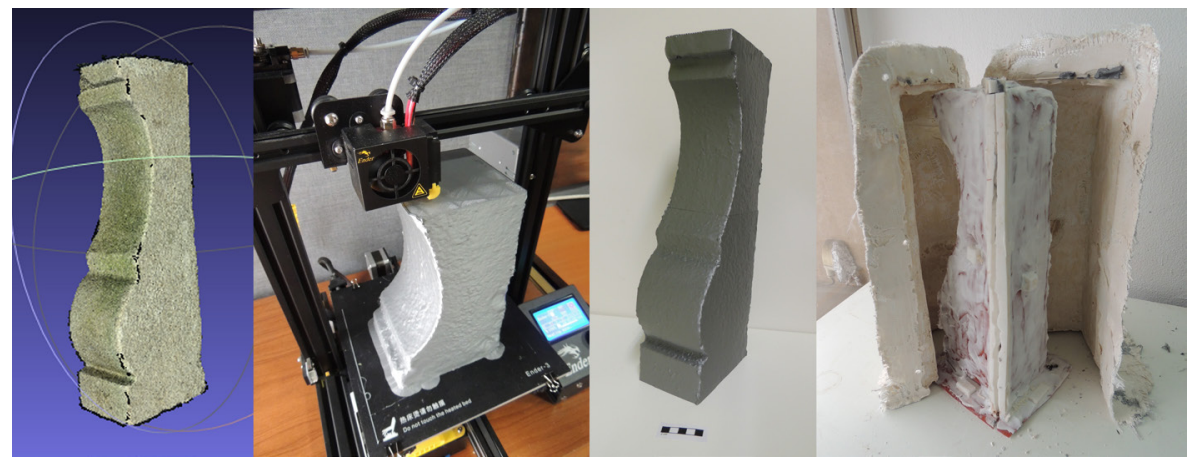

Figura 7: Proceso de realización de un molde laminado indirecto para el balaustre del modelo B

Fuente: Grupo B.B.A.A. (2020) 


\subsection{Molde laminado para la inclusión de frag- mentos originales}

En cuanto al modelo C, primero se estudió con detenimiento la geometría de la pieza. Al no conocerse el aspecto de la zona central del balaustre, se optó por realizar un fuste liso que permitiera la reconstrucción de la balaustrada y la correcta comprensión del espacio, pero sin añadir elementos cuyo aspecto real ignoramos.

Una vez conocida la geometría final que se le iba a dar a la pieza, se preparó una terraja. Esta técnica consiste en una plancha metálica recortada, según el perfil de la pieza deseada, que se incorpora al conjunto y que actúa como generatriz. Se encastra la citada plancha metálica, dentro de una caja de madera, la cual cuenta con un eje, sobre el que se va aplicando el yeso. Este, al girar, adquiere una forma que corresponde al negativo de la plancha (Chavarria, 2003)

Una vez asimilada de manera teórica la realización de modelo, se procedió al dibujado la silueta del balaustre sobre una plancha metálica; se eliminó la parte que correspondería al positivo (esta pieza puede ser reforzada con una plancha de madera, si es necesario); la silueta ya recortada y limada se coloca en una caja preparada previamente, con un eje en la parte central (al mencionado eje se le suelen añadir pasadores, cuerda, o esparto para mejorar el agarre); a continuación, se comienza a verter el yeso, lentamente, sobre el citado eje, mientras se hace rotar el mismo. Conforme crece el diámetro de la pieza, esta va adquiriendo la forma deseada (Chavarria, 2003; Navarro, 2011). Una vez endurecido el yeso se extrae de la terraja y se corta el eje central del prototipo del balaustre. Después, se lija la superficie y se corrige la forma de la base (Figura 8).

En este punto se realiza un molde laminado de silicona, tal y como se describe para el modelo A, para lo que se utiliza como base el prototipo que se ha creado en yeso. Aunque la técnica básica es la misma, en este caso se han incluido aberturas en los dos extremos, y las capas de silicona presentan un grosor mucho mayor. Estas modificaciones tienen por objetivo permitir la colocación de los fragmentos originales dentro del molde, quedando finalmente incorporados en las piezas nuevas. Para mejorar la capacidad elástica del citado molde se optó por incluir en las dos capas más exteriores trozos de silicona de moldes desechados (si no se cuenta con este material podrían añadir más capas de silicona) hasta obtener un grosor considerable.

A la hora de positivar la pieza colocamos dentro del molde, en su posición, uno de los fragmentos recuperados; posteriormente se rellena el molde quedando el original incorporado dentro de la nueva pieza.

\section{Resultados}

Los tres modelos estudiados en este trabajo presentan diferentes estados de conservación: el modelo A cuenta con algunos daños, pero se conserva en su gran mayoría; el modelo B se encuentra seriamente dañado. No obstante, se han conservado los restos suficientes para conocer con detalle su disposición; en cuanto al modelo $\mathrm{C}$, se encuentra muy dañado, desconociéndose actualmente algunos elementos de su diseño. Esto va a afectar también a la manera en la que se plantea su restauración.

La fractura y perdida de elementos ha sido sin duda la degradación más grave que han sufrido las balaustradas de Punta Begoña, habiéndose producido en algunos puntos por motivos intrínsecos, problemas en el diseño o colocación; y, en otros, por agentes extrínsecos, principalmente la mano del hombre.

Como se ha dicho anteriormente, en el modelo A, pese a ser el mejor conservado, aparecen perdidas de los balaustres por todo el conjunto y en zonas puntuales de los barandales superior e inferior. De los 1831 balaustres originales que se han contabilizado, actualmente faltan 313 (Figura 9) (Bermejo, 2019). Fuera de su posición, durante las tareas de desescombro se recuperaron 50 balaustres completos, así como unos 130 fragmentos que se pretenden recolocar cuando se aborde la restauración completa.

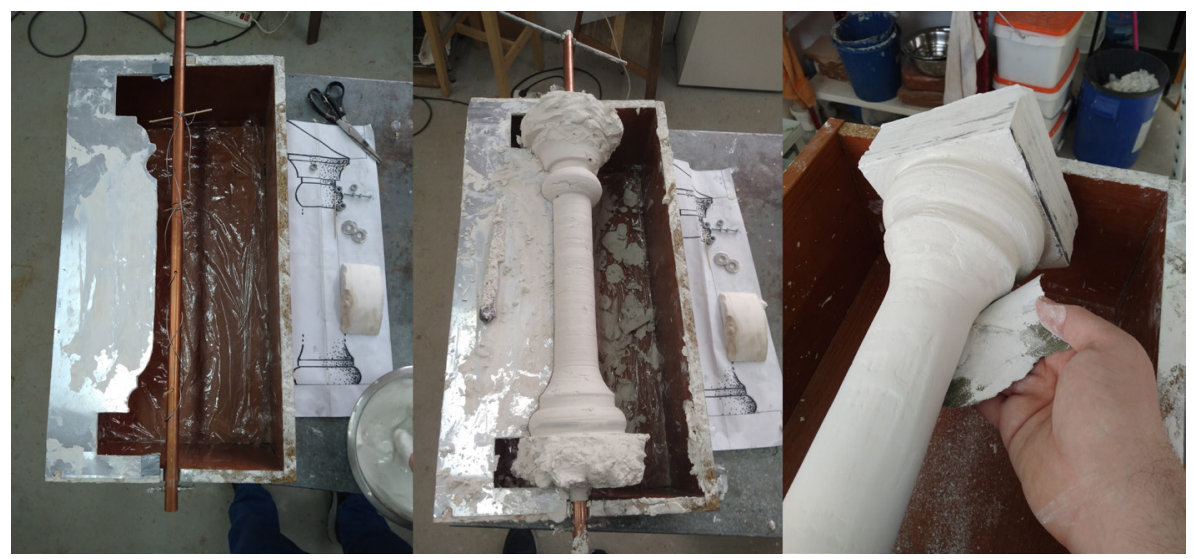

Figura 8: Proceso de realización del prototipo para el balaustre del modelo C con la ayuda de una terraja Fuente: ACC Escultura (2020) 

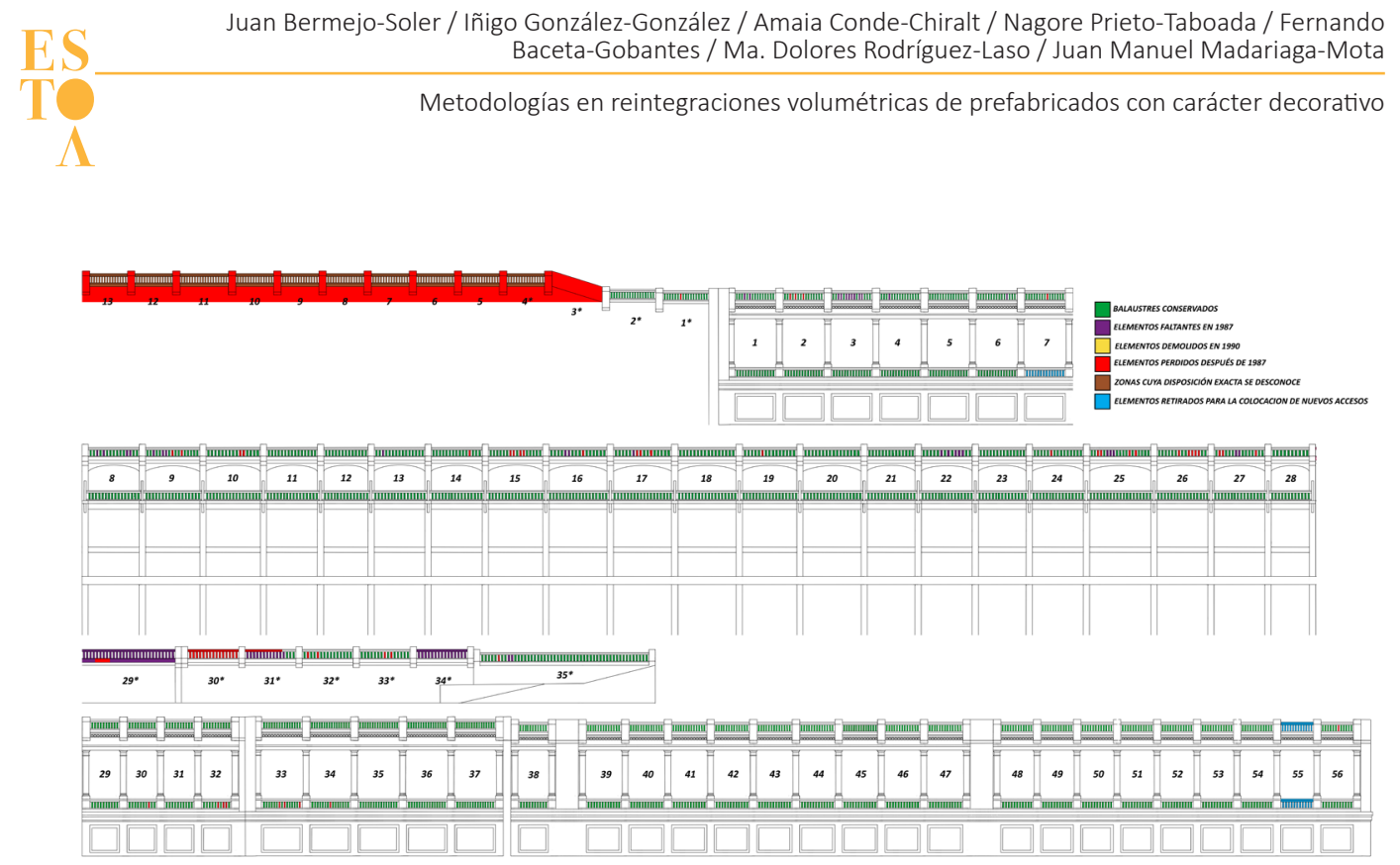

Figura 9: Mapeo de los elementos faltantes del modelo A Fuente: Grupo B.B.A.A. (2019)

El modelo B ha sufrido grandes daños, por lo que quedan en su posición original solo los barandales inferiores. Se han encontrado 22 fragmentos del barandal superior, correspondiente al $60 \%$ del total, en algunos de los cuales se ha podido conocer su posición original por las marcas de unión entre ellos. Se ha hallado un único balaustre completo y 7 fragmentos, de los 58 que lo formaban originalmente (Figura 10). las características de su conservación: para el modelo A se optó por la realización de un molde laminado convencional, en el que se utilizó como base uno de los balaustres completos, encontrados fuera de lugar; en el modelo B, al quedar solo un balaustre original, se prefirió optar por un método indirecto para preservar la pieza al máximo; por último, en el modelo $C$, fue necesario preparar un prototipo con la información recogida de

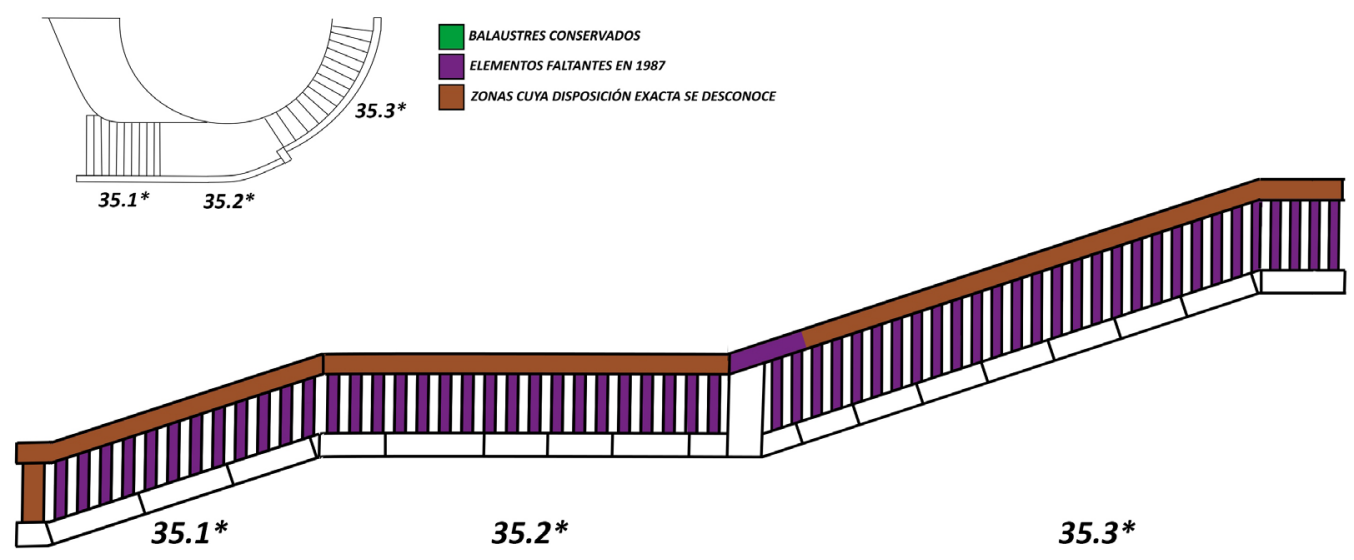

Figura 10: Mapeo de los elementos faltantes del modelo B Fuente: Grupo B.B.A.A. (2019)

El modelo C se encuentra en la zona de acceso a la galería SW. Al comienzo de la puesta en valor de las Galerías Punta Begoña en 2013, esta parte del conjunto arquitectónico se encontraba parcialmente cubierta por tierra y escombros, por lo que, en su posición original, solo quedaba un módulo del barandal superior y todos los barandales inferiores.

La limpieza de esta zona, realizada en 2018, dejó al descubierto 11 fragmentos de los balaustres, 8 fragmentos del barandal superior y los dos bloques prefabricados de hormigón que servían de postes en las esquinas del tramo inferior.

Para la reintegración volumétrica de cada uno de los modelos se propuso la opción que más se adapta a

los fragmentos, y sobre él realizar un molde en el que se pudieran incluir los distintos restos encontrados. Este sistema de moldeo es más complejo que los anteriores, pero permite incorporar al máximo los fragmentos originales, a la par de agilizar enormemente los procesos de restauración.

\section{Conclusiones}

La realización de reproducciones es muchas veces necesaria cuando se trata de intervenciones en patrimonio construido, ya que en muchas ocasiones los elementos prefabricados tienen una funcionalidad de cerramiento o soporte de otros materiales, además de la parte estética. No obstante, la reintegración de estos ha de hacerse con unos criterios adecuados, que permitan 
una correcta lectura de la obra, pero respetando al máximo posible los materiales originales que se han conservado. En muchas ocasiones actualmente esto no es así y cualquier prefabricado dañado o fragmentado es sustituido por uno nuevo.

Con este trabajo se pretende dar a conocer opciones como la inclusión de fragmentos originales dentro de propio molde o la realización de moldes indirectos con apoyo de la tecnología 3D, para ayudar a la conservación de este tipo de elementos con medidas alcanzables en la mayoría de los proyectos de rehabilitación.

\section{Agradecimientos}

El siguiente trabajo se enmarca en el proyecto de "Recuperación y puesta en valor de las Galerías Punta Begoña", llevado a cabo a través de la colaboración del Ayuntamiento de Getxo y la Universidad del País Vasco (UPV/EHU). Se busca el desarrollo de nuevas metodologías para la recuperación de los elementos decorativos que forman parte del Patrimonio Construido. Este trabajo está reflejado en diversos artículos, en dos tesis doctorales ya defendidas, la primera sobre azulejos (Madariaga, 2018) y la segunda sobre pinturas murales (Lama, 2020), además de otros dos proyectos de tesis aun en marcha.

Cómo citar este artículo/How to cite this article: Bermejo-Soler, J., González-González, I., Conde-Chiralt, A., Prieto-Taboada, N., Baceta-Gobantes, F., Rodríguez-Laso,M.D. y Madariaga-Mota, J.M. (2022). Metodologías en reintegraciones volumétricas de prefabricados con carácter decorativo. Estoa. Revista de la Facultad de Arquitectura y Urbanismo de la Universidad de Cuenca, 11(21), 117-125. https:// doi.org/10.18537/est.v011.n021.a10

\section{Referencias bibliográficas}

Arroitia, G., Peraita, M. y Amezaga, J. (2020). Las 150 vidas de Horacio Echevarrieta. Surfing Challenge.

Bermejo, J. (2019) Las balaustradas como elementos de piedra artificial en las galerías Punta Begoña (Getxo). Estudio y caracterización [Trabajo de final de máster, UPV/EHU].

Bermejo, J., Solano, K., Lama, E., Madariaga, I., Venegas, C., Baceta, F. y Rodríguez, M.D. (2018). La seguridad como aval de la conservación preventiva de las Galerías de Punta Begoña. VI Congreso GEIIC: ¿YY después? Control y mantenimiento del Patrimonio Cultural, una opción sostenible (pp. 462-467). International Institute for Conservation of Historic and Artistic Works.

Burgos, A. (2009). Los orígenes del hormigón armado en España. Ministerio de Fomento.

Calvo, A. (2016). Estudio comparativo de las galerías miradores del siglo XX de Euskadi [Trabajo de final de máster, UPV/EHU].

Chavarria, J. (2003). Moldes. Paramón.

Gondra, J. [Galerías de Punta Begoña (Getxo, Bizkaia)]. (22 de marzo de 2018). Píldora documental 'Sanidad militar' [Archivo de video]. https://www.youtube.com/ watch?v=0BB2pGrSsGo

Lama, E., (2020). Desarrollo de un protocolo integral para la puesta en valor de los elementos decorativos del salón de las galerías Punta Begoña (Getxo) [tesis doctoral, UPV/ EHU].

Madariaga, I. (2018). Diseño de protocolos de actuación para la eliminación de materiales acumulados sobre las piezas cerámicas de las galerías Punta Begoña en Getxo [tesis doctoral, UPV/EHU].

Mas, X. (2006). Estudio y caracterización de morteros compuestos, para su aplicación en intervenciones de sellados, reposiciones y réplicas, de elementos pétreos escultóricos - ornamentales [tesis doctoral, Universitat Politècnica de València].

Navarro, J.L. (2011). Maquetas, modelos y moldes: Materiales y técnicas para dar forma a las ideas. Publicaciones de la Universitat Jaume I. 